\title{
Structure of fuzzy dot BF-subalgebras
}

\author{
Gerima Tefera \\ Dept. Mathematics (of Aff.) \\ Wollo University (of Aff.) \\ Dessie, Ethiopia \\ email gerima233@gmail.com
}

\author{
Tigist Fantahun \\ Dept. Mathematics (of Aff.) \\ Wollo University (of Aff.) \\ Dessie, Ethiopia \\ email tigf91@gmail.com
}

\begin{abstract}
The concept of fuzzy dot $B F$-subalgebra has been introduced. Fuzzy dot product of $B F-$ subalgebras and strong fuzy product in a fuzzy dot $B F-$ subalgebra has been discused.

Different theorems,Lemmas and propositions has been proved. We have also investigated different characterizations.

Index Terms-BF- subalgebra,fuzzy BFsubalgebras, Ideal in BF- subalgebras, Fuzzy dot BF-subalgebras.
\end{abstract}

\section{INTRODUCTION}

In [8] the idea of fuzzy set was first introduced by Zadeh and the concept of B-algebras was introduced by Neggers and Kim. They defined a B-algebra as an algebra $(X, *, 0)$ of type $(2,0)$ satisfying the following axioms:

1) $x * x=0$.

2) $x * 0=x$.

3) $(x * y) * z=x *(z *(0 * y))$.

Andrzej Walendziak initaited the idea of a BF-algebra and characterized different structures in [1] and in [3] Borumand Saeid and Rezrani investigated Fuzzy BFAlgebras.

In this paper, we introduced the concept of fuzzy dot BF-algebras and study its structures. We state and prove some theorem discussed in fuzzy dot BFsubalgebras and level subalgebras. Finally some of results on homomorphic images and inverse images in fuzzy dot BF-subalgebras are investigated.

\section{PRELIMINARIES}

A BF-algebra is a nonempty set $X$ with a constant ' 0 ' and a binary operation ' $*$ ' satisfying the following conditions:

1) $x * x=0$.

2) $x * 0=x$.

3) $0 *(x * y)=(y * x)$ For all $x, y \in X$.

Example 2.1: Let $X=[0, \infty)$. Define the binary operation ' $*$ ' on $X$ as follows: $x * y=|x-y|$, for all $x, y \in X$, Then $(X, *, 0)$ is a BF-algebra.
Let $X$ be a BF-algebra. Then for any $x$ and $y$ in $X$, the following hold:

1) $0 *(0 * x)=x$.

2) if $0 * x=0 * y$, then $x=y$.

$3)$ if $x * y=0$,then $y * x=0$.

Any BF-algebra $(X, *, 0)$ that satisfies the identity $(x * z) *(y * z)=x * y$ is a B-algebra. A subset $I$ of $X$ is called an ideal of $X$ if it satisfies:

1) $0 \in I$.

2) $x * y$ and $y \in I$ imply $x \in I$ for any $x, y \in X$.

A nonempty subset $S$ of $X$ is called a sub algebra of $A$ if $x * y \in S$ for any $x, y \in X$.

Let $\left(X, *, 0_{X}\right)$ and $\left(Y, *, 0_{Y}\right)$ be BF-algebras. A mapping $\phi: X \longrightarrow Y$ is called a homomorphism from $X$ into $Y$ if $\phi(x * y)=\phi(x) * \phi(y)$ for any $x, y \in X$.

Let $X$ be a nonempty set. A fuzzy (sub) set $\mu$ of the set $X$ is a mapping $\mu: X \longrightarrow[0,1]$.

and $\mu$ is the fuzzy set of a set $X$. For a fixed $s \in[0,1]$, the set $\mu_{s}=\{x \in X: \mu(x) \geq s\}$ is called an upper level of $\mu$ or level subset of $\mu$. Furthermore let $X$ be a set. A fuzzy set $A$ in $X$ is characterized by a membership function $\mu_{A}: X \longrightarrow[0,1]$. Let $f$ be a mapping from the set $X$ to the set $Y$ and let $B$ be a fuzzy set in $Y$ with membership function $\mu_{B}$.

The inverse image of $B$, denoted $f^{-1}(B)$, is the fuzzy set in $X$ with membership function $\mu_{f^{-1} B}$ defined by $\mu_{f^{-1}(B)(x)}=\mu_{B}(f(x))$ for all $x \in X$. Conversely, let $A$ be a fuzzy set in $X$ with membership function $\mu_{A}$ Then the image of $A$, denoted by $f(A)$, is the fuzzy set in $Y$ such that $\mu_{f(A)}(y)=$ $\left\{\begin{array}{ll}\sup \mu_{A}(x)_{x \in f^{-1}(y)} & \text { if } f^{-1}(y)=\{x: f(x(=y\} \\ 0 & \text { if otherwise }\end{array}\right.$. A fuzzy set $A$ in the BF -algebra $X$ with the membership function $\mu_{A}$ is said to be have the sup property if for any subset $T \subseteq X$ there exists $x_{0} \in T$ such that $\mu_{A}\left(x_{0}\right)=\sup \mu_{A}(t)_{t \in T}$; and let $\mu$ be a fuzzy set in a $\mathrm{BF}$-algebra. Then $\mu$ is called a fuzzy BF -subalgebra (algebra) of $X$ if $\mu(x * y) \geq \min \{\mu(x), \mu(y)\}$ for all $x, y \in X$. 
A fuzzy set $\mu$ of a BF-algebra $X$ is called a fuzzy ideal of $X$ if it satisfies the following conditions.

1) $\mu(0) \geq \mu(x)$

2) $\mu(x) \geq \min \{\mu(x * y), \mu(y)\}$

\section{RESULTS}

\section{A. Fuzzy dot BF-Sub algebra}

Definition 3.1: Let A be a fuzzy set in BFSubalgebra $\mathrm{X}$ and $\mu$ be a fuzzy subset a BF-Subalgebra of $\mathrm{X}$. Then the fuzzy dot BF-Subalgebra of $\mathrm{X}$ is $\mu_{A}(x \star y) \geq \mu_{A}(x) \cdot \mu_{A}(y)$, for all $x, y \in X$.

Example 3.2: Let $X=\{0, a, b\}$ be a set with the table given below

\begin{tabular}{|c|c|c|c|}
\hline$\star$ & 0 & $\mathrm{a}$ & $\mathrm{b}$ \\
\hline 0 & 0 & $\mathrm{a}$ & $\mathrm{b}$ \\
\hline $\mathrm{a}$ & $\mathrm{a}$ & 0 & 0 \\
\hline $\mathrm{b}$ & $\mathrm{b}$ & 0 & 0 \\
\hline
\end{tabular}

. Then $(X, \star, 0)$ is a $B F-$ algebra.

Define $\mu: X \rightarrow[0,1]$ by $\mu(0)=0.8, \mu(a)=0.2$, and $\mu(b)=0.4$. Then $\mu$ is a fuzzy dot $B F-$ Subalgebra of X. Since $\mu(a \star b)=\mu(0)=0.8 \geq(0.2)(0.4)=$ $0.08=\mu(a) \cdot \mu(b)$.

Hence $\mu(a \star b) \geq \mu(a) . \mu(b)$, for all $a, b \in X$.

Lemma 3.3: Let A be a fuzzy set in $B F$ - algebra. If $\mu_{A}$ is a fuzzy dot BF-Subalgebra of $\mathrm{X}$, then for all $x \in X, \mu(0) \geq(\mu(x))^{2}$, for all $x \in X$.

Corollary 3.1: If $\mathrm{A}$ is a fuzzy subset of a BF-algebra $\mathrm{X}$ and $\mu_{A}$ is a a fuzzy dot subalgebra of $\mathrm{X}$, then $\mu_{A}\left(0^{n} \star x\right) \geq$ $\left(\mu_{A}(x)\right)^{2 n+1}$, for all $x \in X$ and $n \in N$, where $0^{n} \star x=0 \star(0 \star(0 \star \ldots(0 \star x) \ldots)$ in which 0 occurs $n$-times.

Proof. By Lemma 3.3. we have $\mu_{A}(0) \geq$ $\left(\mu_{A}(x)\right)^{2}$, for all $x \in X$. Put $\mathrm{n}=1$ in $0^{n} \star a$ we have $0 \star x$.

$\mu_{A}(0 \star x) \geq \mu_{A}(0) \cdot \mu_{A}(x) \geq\left(\mu_{A}(x)\right)^{2} \cdot \mu_{A}(x)=$ $\left(\mu_{A}(x)\right)^{3}$.

Hence $\mu_{A}(0 \star x) \geq\left(\mu_{A}(x)\right)^{3}$, for all $x \in X$.

Assume the result holds for $\mathrm{n}=\mathrm{k}, \mu_{A}\left(0^{k} \star x\right) \geq$ $\left(\mu_{A}(x)\right)^{2 k+1}$, for all $x \in X$.

$$
\begin{array}{r}
\text { Now } \mu_{A}\left(0^{k+1} \star x\right)=\mu_{A}\left(0 \star\left(0^{k} \star x\right)\right) \\
\geq \mu(0) \cdot \mu\left(o^{k} \star x\right) \\
\geq\left(\mu_{A}(x)\right)^{2} \cdot\left(\mu_{A}(x)\right)^{2 k+1} \\
=\left(\mu_{A}(x)\right)^{2(k+1)+1}
\end{array}
$$

Hence $\quad \mu_{A}\left(0^{n} \quad \star \quad x\right)$

$\left(\mu_{A}(x)\right)^{2 n+1}$, for all $x \in X$ and $n \in N . \square$

Theorem 3.2: Let A be a fuzzy subset of $B F$-Subalgebra and let $\mu_{A}$ be a fuzzy dot BFSubalgebra of $X$. If there exists a sequence $\left\{x_{n}\right\}$ in $\mathrm{X}$ such that $\operatorname{liim}_{n \rightarrow \infty} \mu_{A}\left(x_{n}\right)=1$. then $\mu_{A}(0)=1$.
Theorem 3.3: Let $A_{1}$ and $A_{1}$ be fuzzy subsets of $B F$-Subalgebras and let $\mu_{A_{1}}$ and $\mu_{A_{2}}$ be fuzzy dot $B F$ - Subalgebras of $\mathrm{X}$. Then $\mu_{A_{1} \cap A_{1}}$ is a fuzzy dot subalgebras of $\mathrm{X}$.

Proof. Let $x, y \in A_{1} \cap A_{2}$. Then $x, y \in A_{1}$ and $x, y \in A_{2}$, since $A_{1}$ and $A_{2}$ are subsets of fuzzy $B F$-Subalgebra of X.

$\mu_{A_{1} \cap A_{2}}(x \star y)=\min \left\{\mu_{A_{1}}(x \star y), \mu_{A_{2}}(x \star y)\right\}$

$\geq \min \left\{\mu_{A_{1}}(x) \cdot \mu_{A_{1}}(x), \mu_{A_{2}}(x) \cdot \mu_{A_{2}}(y)\right\}$

$\geq \mu_{A_{1} \cap A_{2}}(x) \cdot \mu_{A_{1} \cap A_{2}}(y)$.

Hence $\mu_{A_{1} \cap A_{2}}(x \star y)$

$\geq \min \left\{\min \left\{\mu_{A_{1}}(x), \mu_{A_{2}}(x)\right\} \cdot \min \left\{\mu_{A_{1}}(y), \mu_{A_{2}}(y)\right\}\right\} \geq$ $\mu_{A_{1} \cap A_{2}}(x), \mu_{A_{1} \cap A_{2}}(y)$.

Corollary 3.4: Let $\left\{A_{i} \mid i \in I\right\}$ be a family of fuzzy dot $B F$-Subalgebras of $\mathrm{X}$. Then $\cap_{i \in I} A_{i}$ is also a fuzzy dot $B F$-Subalgebras of $\mathrm{X}$.

Proof. Let $\left\{A_{i} \mid i \in I\right\}$ be a family of fuzzy dot $B F-$ Subalgebra. Then $\mu_{A_{i}}$ is a fuzzy dot $B F-$ Subalgebra of $\mathrm{X}$ for each $i \in I$.

We have to show $\mu_{\cap_{i \in I} A_{i}}$ is a fuzzy dot $B F$-Subalgebra of X.

Consider $\quad \mu_{\cap_{i \in I} A_{i}}(a \quad \star \quad b)=$

$\min \left\{\mu_{A_{i}}(x \star y), \mu_{A_{j}}(x \star y)\right\}$

$\geq \min \left\{\mu_{A_{i}}(x) \cdot \mu_{A_{i}}(y), \mu_{A_{j}}(x) \cdot \mu_{A_{j}}(y)\right\}$

$\geq \min \left\{\min \left\{\mu_{A_{i}}(x), \mu_{A_{j}}(x)\right\} \cdot \min \left\{\mu_{A_{i}}(y), \mu_{A_{j}}(y)\right\}\right\}$

$\geq \mu_{\cap_{i \in I} A_{i}}(x) \cdot \mu_{\cap_{i \in I} A_{i}}(y)$.

Hence $\mu_{\cap_{i \in I} A_{i}}(x \star y) \geq \mu_{\cap_{i \in I} A_{i}}(x) \cdot \mu_{\cap_{i \in I} A_{i}}(y)$.

Definition 3.5: Let $\mathrm{A}$ be a fuzzy set in $\mathrm{X}$ and $\theta \in[0,1]$. Then the level $B F-$ Subalgebra of $U(A, \theta)$ of A and strong level $B F$-Subalgebra $U(A,>, \theta)$ of $\mathrm{X}$ are defined as follows:

$U(A, \theta)=\left\{x \in X \mid \mu_{A}(x) \geq \theta\right\}$.

$U(A,>, \theta)=\left\{x \in X \mid \mu_{A}(x)>\theta\right\}$.

\section{Theorem 3.4:}

Let $A$ be a non-empty subset of $X$.Then $A$ is sub algebra of $X$ if and only if $X_{A}$ is fuzzy dot sub algebra of $X$.

Proof. Let $A$ be sub algebra of $X$ and $x, y \in A$.Then $x, y \in A$, Then we have

$X_{A}(x * y) \geq X_{A}(x) \cdot X_{A}(y)$

since $1 \geq(1) .(1)=1 \geq 1$, because $x, y \in A$ then $x * y \in A$

If $x \in A$ and $y \notin A$ then we get $X_{A}(x)=1$ or $X_{A}(y)=0$

$X_{A}(x * y) \geq \mu_{A}(x) \cdot \mu_{A}(y)$

$1 \geq(1) .(0)=1 \geq 0$, because $A$ is subset of $X$ and $x, y \in X$.

If $x \notin A$ and $y \in A$ then we get $X_{A}(x)=0$ or $X_{A}(y)=1$

$X_{A}(x * y) \geq X_{A}(x) \cdot X_{A}(y)$

$1 \geq(0)$.(1) $\geq 1$, because $A$ is subset of $X$ and $x \in X$. Conversely that $X_{A}$ is a fuzzy dot sub algebra of $X$ and let $x, y \in A$. Then

$X_{A}(x * y) \geq X_{A}(x) \cdot X_{A}(y)$ 
$1 \geq X_{A}(x * y) \geq 1$

$X_{A}(x * y)=1$. Then , $x * y \in A$. Hence $A$ is a sub algebra of $\mathrm{X} . \square$

Theorem 3.5: Let A be a fuzzy set in a fuzzy $B F-$ Sub algebra of $\mathrm{X}$ and $\mu_{A}$ be a fuzzy dot $B F-$ sub algebra of $\mathrm{X}$ with least upper bound $\lambda_{0} \in[0,1]$. Then the following are equivalent.

1) $\mu_{A}$ is a fuzzy dot $B F$-subalgebra of $\mathrm{X}$.

2) For all $\lambda \in \operatorname{Im}\left(\mu_{A}\right)$, the nonempty level subset $U(A, \lambda)$ of $\mathrm{A}$ is a $B F$-subalgebra of $\mathrm{X}$.

3) For all $\lambda \in \operatorname{Im}\left(\mu_{A}\right) / \lambda_{0}$, the non empty strong level subset $U(A,>, \lambda)$ of $\mathrm{A}$ is a $B F$ - subalgebra of $X$.

4) For all $\lambda \in[0,1]$, the nonempty strong level subset $U(A, \geq, \lambda)$ of $\mathrm{A}$ is a $B F$-subalgebra of $\mathrm{X}$.

5) For all $\lambda \in[0,1]$, the nonempty level subset $U(A, \lambda)$ of $\mathrm{A}$ is a $B F-$ sub algebra of $\mathrm{X}$.

\section{Proof.}

1) $1 \Rightarrow 4$, let $\mathrm{A}$ be a fuzzy set in a $B F$-sub algebra and $\mu_{A}$ be a fuzzy dot $B F-$ Sub algebra of $\mathrm{X}, A \in[0,1]$ and let $x, y \in U(A,>, \lambda)$. Then $\mu_{A}(x \star y) \geq \mu_{A}(x) \cdot \mu_{A}(y)>\lambda$. Imply that $x \star y \in U(A,>, \lambda)$.

Hence $U(A,>, \lambda)$ ie a $B F$ - sub algebra of X.

2) $4 \Rightarrow 3$ for each $\lambda \in[0,1], U(A,>, \lambda)$ be a $B F$-sub algebra of $\mathrm{X}$.

Let $\lambda \in I m \mu_{A} / \lambda_{0}$ and $x, y \in U(S,>, \lambda)$. Then $\mu_{A}(a \star b) \geq \mu_{A}(x) \cdot \mu_{A}(y)>\lambda$ by (4).

We have $x \star y \in U(A,>, \lambda)$. Hence $U(A,>, \lambda)$ is a $B F$-sub algebra of $\mathrm{X}$.

3) $3 \Rightarrow 2$ let $\lambda_{0} \in \operatorname{Im}\left(\mu_{A}\right)$. Then $U(A, \lambda)$ is nonempty since $U(A, \lambda)=\cap_{\lambda>\beta} U(A,>, \lambda)$, where $\beta \in \operatorname{Im}\left(\mu_{A}\right) / \lambda_{0}$. Then by (3) $U(A, \lambda)$ is a $B F-$ sub algebra of $X$.

4) $2 \Rightarrow 5$ Let $\lambda \in[0,1]$ and $U(A, \lambda)$ be nonempty. Suppose $x, y \in U(A, \lambda)$ and let $\alpha=\min \left\{\mu_{A}(x), \mu_{A}(y)\right\}$, since $\mu_{A}(x) \geq \lambda$ and $\mu_{A}>\lambda$. Imply that $\alpha \geq \lambda$, where $\lambda=$ $\mu_{A}(x) \cdot \mu_{A}(y)$.

Thus $x, y \in U(A, \alpha)$ and $\alpha \in I m \mu_{A}$ by 2 $U(A, \alpha)$ is a $B F-$ sub algebra of $\mathrm{X}$. Hence $\mu_{A}(x \star y) \geq \mu_{A}(x) \cdot \mu_{A}(y)=\lambda$.

Thus $x \star y \in U(A, \alpha)$. Then we have $x \star y \in$ $U(A, \lambda)$. Hence $U(A, \lambda)$ is a $B F$-sub algebra of $\mathrm{X}$.

5) Assume that the non-empty set $U(A, \lambda)$ is a $B F$-sub algebra of $\mathrm{X}$ for every $\lambda \in[0,1]$.

Suppose $x_{0}, y_{0} \in X$ such that $\mu_{A}\left(x_{0} \star y_{0}<\right.$ $\mu_{A}\left(x_{0}\right) \cdot \mu_{A}\left(y_{0}\right)$. Put $\mu_{A}\left(x_{0}\right)=\beta, \mu_{A}\left(y_{0}\right)=\delta$ and $\mu_{A}\left(x_{0} \star y_{0}\right)=\theta$. Then $\theta<\min \{\beta, \delta\}$.

Consider $\theta_{1}=\frac{1}{2}\left(\mu_{A}\left(x_{0} \star y_{0}\right)+\mu_{A}\left(x_{0}\right) \cdot \mu_{A}\left(y_{0}\right)\right.$, we get $\theta_{1}=\frac{1}{2}(\theta+\beta . \delta)$.

Therefore $\beta>\theta_{1}=\frac{1}{2}(\theta+\beta . \delta)>\theta$. $\delta>\theta_{1}=\frac{1}{2}(\theta+\beta . \delta)>\theta$.

Hence $\beta . \delta>\theta_{1}>\theta=\mu_{A}\left(x_{0} \star y_{0}\right)$.

Hence $x_{0} \star y_{0} \notin U(A, \theta)$ which is a contradiction since $\mu_{A}\left(x_{0}\right)=\beta \geq \beta . \delta>\theta_{1}$.

$\mu_{A}\left(b_{0}\right)=\delta \geq \beta . \delta>\theta_{1}$. Imply that $x_{0}, y_{0} \in U(A, \theta)$.

Thus $\quad \mu_{A}(x \quad \star \quad y) \quad \geq$ completes the proof.

Theorem 3.6: Each BF- Sub algebra of $\mathrm{X}$ is a level $B F$ - sub algebra of a fuzzy dot $B F$ - sub algebra of $\mathrm{X}$.

Proof Let $\mathrm{B}$ be a $B F-$ sub algebra of $\mathrm{X}$ and $\mathrm{A}$ be any fuzzy set in $\mathrm{X}$ defined by $\mu_{A}(x)= \begin{cases}\gamma & \text { if } x \in B \\ 0 & \text { if } x \notin B\end{cases}$ , where $\gamma \in[0,1]$.

Let $y \in U(A, \gamma)$. Then $\mu_{A}(y) \geq \gamma$, for all $y \in A$. So that $y \in B$.

Hence $U(A, \gamma) \subseteq B$.

Let $z \in B$. Then $\mu_{A}(z)=\gamma$. Imply that $\mu_{A}(z) \geq \gamma$. Hence $z \in U(A, \gamma)$.

Thus $B \subseteq U(A, \gamma)$. It follows that $U(A, \gamma)=B$.

We consider the following cases:

1) If $x, y \in B$, then $\mu_{A}(x \star y)=\gamma$ $\gamma \cdot \gamma=\gamma^{2}=\mu_{A}(x) \cdot \mu_{A}(y)$. Hence $x \star y \in B$.

2) If $x, y \notin B$, then $\mu_{A}(x)=0=\mu_{A}(y)$. So that $\mu_{A}(x \star y) \geq 0=\min \{0,0\}=$ $\min \left\{\mu_{A}(x), \mu_{A}(y)\right\} \geq \mu_{A}(x) \cdot \mu_{A}(y)$.

Hence $\mu_{A}(x \star y) \geq \mu_{A}(x) . \mu_{A}(y)$.

3) If $x \in B$ and $y \notin B$, then $\mu_{A}(x)=\gamma$ and $\mu_{A}(y)=0$.

Thus $\mu_{A}(x \star y) \geq 0=\min \{\gamma, 0\}=$ $\min \left\{\mu_{A}(x), \mu_{A}(y)\right\}$

$\geq \mu_{A}(x) \cdot \mu_{A}(y)$.

Hence $\mu_{A}(x \star y) \geq \mu_{A}(x) \cdot \mu_{A}(y)$.

4) If $y \in B$ and $x \notin B$, then by the same argument as in case 3 , we can conclude that $\mu_{A}(x \star y) \geq$ $0=\min \{0, \gamma\}=\min \left\{\mu_{A}(x), \mu_{A}(y)\right\}$

$\geq \mu_{A}(x) \cdot \mu_{A}(y)$. Hence $\mu_{A}$ is a fuzzy dot $B F-$ subalgebra of X.

Theorem 3.7: Let B be a subset of $\mathrm{X}$ and $\mathrm{A}$ be a fuzzy set on $\mathrm{X}$ which is given by the proof of theorem 5.6 If $\mu_{A}$ is a fuzzy dot $B F$-sub algebra of $\mathrm{X}$, then $\mathrm{B}$ is a $B F$-sub algebra of $\mathrm{X}$.

Theorem 3.8: Let A be a fuzzy set in $B F-$ sub algebra X. If $\mu_{A}$ is a fuzzy dot $B F$-sub algebra of $\mathrm{X}$, then the set $X_{\mu_{A}}=\left\{x \in X / \mu_{A}(x)=\mu_{A}(0)\right\}$ is a $B F-$ sub algebra of $X$.

Proof. Let $x, y \in X_{\mu_{A}}$. Then $\mu_{A}(x)=\mu_{A}(0)=$ $\mu_{A}(y)$ and so $\mu_{A}(x \star y) \geq \min \left\{\mu_{A}(x), \mu_{A}(y)\right\}$

$=\min \left\{\mu_{A}(0), \mu_{A}(0)\right\} \geq \mu_{A}(0) \cdot \mu_{A}(0)$. But $\mu_{A}(0) \geq$ $\mu_{A}(0) . \mu_{A}(0)$. Hence $\mu_{A}(x \star y) \geq \mu_{A}(0)$ and $\mu_{A}(0) \geq$ 
$\mu_{A}(x \star y)$.

Thus $\mu_{A}(x \star y)=\mu_{A}(0)$ which means that $x \star y \in X_{\mu_{A}}$.

Definition 3.6: Let $A=\left\{\left\langle x, \mu_{A}(x)\right\rangle: x \in X\right\}$ and $B=\left\{\left\langle x, \mu_{B}(x)\right\rangle: x \in X\right\}$ be two fuzzy sets in $X$.The Cartesian product $A \times B: X \times X \longrightarrow[0,1]$ is defined by $\left(\mu_{A} \times \mu_{B}\right)(x, y)=\mu_{A}(x) \cdot \mu_{B}(y)$ for all $x, y \in X$.

Proposition 3.7: Let $A$ and $B$ be two fuzzy dot sub algebras of $X$,then $A \times B$ is a fuzzy dot sub algebra of $X \times X$.

Proof. Let $\left(x_{1}, y_{1}\right)$ and $\left(x_{2}, y_{2}\right) \in X \times X$ then $\left(\mu_{A} \times \mu_{B}\right)\left(\left(x_{1}, y_{1}\right) *\left(x_{2}, y_{2}\right)\right)=\left(\mu_{A} \times \mu_{B}\right)\left(\left(x_{1} *\right.\right.$ $\left.x_{2}\right),\left(y_{1} * y_{2}\right)$

$\left(\mu_{A} \times \mu_{B}\right)\left(\left(x_{1} * x_{2}\right),\left(y_{1} * y_{2}\right)=\mu_{A}\left(x_{1} * x_{2}\right) \cdot \mu_{B}\left(y_{1} * y_{2}\right)\right.$

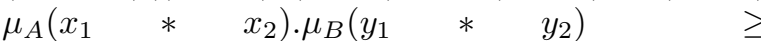
$\left(\mu_{A}\left(x_{1}\right) \cdot \mu_{A}\left(x_{2}\right)\right) \cdot\left(\mu_{B}\left(y_{1}\right) \cdot \mu_{B}\left(y_{2}\right)\right)$ $\left(\mu_{A}\left(x_{1}\right) \cdot \mu_{A}\left(x_{2}\right)\right) \cdot\left(\mu_{B}\left(y_{1}\right) \cdot \mu_{B}\left(y_{2}\right)\right)=\left(\mu_{A} \times\right.$ $\left.\mu_{B}\right)\left(x_{1}, y_{1}\right) \cdot\left(\mu_{A} \times \mu_{B}\right)\left(x_{2}, y_{2}\right)$

Hence, $A \times B$ is fuzzy dot sub algebra of $X \times X$.

Proposition 3.8: Let $A$ and $B$ be two fuzzy dot ideals of $X$, then $A \times B$ is a fuzzy dot ideals of $X \times X$.

Proof. Let $\left(x_{1}, y_{1}\right)$ and $\left(X_{2}, y_{2}\right)$, then

$\left(\mu_{A} \times \mu_{B}\right)\left(x_{1}, y_{1}\right)=\mu_{A}\left(x_{1}\right) \cdot \mu_{B}\left(y_{1}\right)$

$\mu_{A}\left(x_{1}\right) \cdot \mu_{B}\left(y_{1}\right) \geq\left(\mu_{A}\left(x_{1} * x_{2}\right) \cdot \mu_{A}\left(x_{2}\right)\right) \cdot\left(\mu_{B}\left(y_{1} *\right.\right.$ $\left.\left.y_{2}\right) \cdot \mu_{B}\left(y_{2}\right)\right)$

$=\left(\mu_{A}\left(x_{1} * x_{2}\right) \cdot \mu_{B}\left(y_{1} * y_{2}\right)\right) \cdot\left(\mu_{A}\left(x_{2}\right) \cdot \mu_{B}\left(y_{2}\right)\right)$

$=\left(\mu_{A} \times \mu_{B}\right)\left(x_{1} * x_{2}, y_{1} * y_{2}\right) \cdot\left(\mu_{A} \times \mu_{B}\right)\left(x_{2}, y_{2}\right)$

$=\left(\mu_{A} \times \mu_{B}\right)\left(\left(x_{1}, y_{1}\right) *\left(x_{2}, y_{2}\right)\right) \cdot\left(\mu_{A} \times \mu_{B}\right)\left(x_{2}, y_{2}\right)$

Hence $A \times B$ is a fuzzy dot ideal of $X \times X$.

\section{B. Fuzzy $\rho$-dot product Relation of BF-Algebra}

In this section, strongest fuzzy $\rho$-relation and fuzzy $\rho$-product relation of BF-algebras are defined and presented some of its properties.

Definition 3.9: Let $\rho$ be a fuzzy sub set of $X$. The strongest fuzzy $\rho$-relation on $X$ is the fuzzy subset $\mu_{\rho}$ of $X \times X$ given by $\mu_{\rho}(x, y)=\rho(x) . \rho(y)$ for $x, y \in X$

Theorem 3.9: Let $\mu_{\rho}$ be the strongest fuzzy $\rho$ relation on $X$, where $\rho$ is a sub set of $X$, If $\rho$ is a fuzzy dot sub algebra of $X$, then $\mu_{\rho}$ is a fuzzy dot sub algebra of $X \times X$.

Proof. Suppose that $\rho$ is fuzzy dot sub algebra of $X$. For any $\left(x_{1}, y_{1}\right)$ and $\left(x_{2}, y_{2}\right) \in X \times X$

We have $\left(\mu_{\rho}\left(x_{1}, y_{1}\right) *\left(x_{2}, y_{2}\right)\right)=\mu_{\rho}\left(x_{1} * x_{2}\right) \cdot \mu_{\rho}\left(y_{1} *\right.$ $\left.y_{2}\right)$

By the definition of strongest fuzzy $\rho$-relation of BF-algebras we get

$m u_{\rho}\left(x_{1} * x_{2}\right) \cdot \mu_{\rho}\left(y_{1} * y_{2}\right)=\rho\left(x_{1} * x_{2}\right) \cdot \rho\left(y_{1} * y_{2}\right)$

$\rho\left(x_{1} * x_{2}\right) \cdot \rho\left(y_{1} * y_{2}\right) \geq \rho\left(x_{1}\right) \cdot \rho\left(x_{2}\right) \cdot \rho\left(y_{1}\right) \cdot \rho\left(y_{2}\right)$ (since a fuzzy dot sub algebra of)

$\rho\left(x_{1}\right) \cdot \rho\left(x_{2}\right) \cdot \rho\left(y_{1}\right) \cdot \rho\left(y_{2}\right)$

$\left(\rho\left(x_{1}\right) \cdot \rho\left(y_{1}\right)\right) \cdot\left(\rho\left(x_{2}\right) \cdot \rho\left(y_{2}\right)\right)$ $\left(\rho\left(x_{1}\right) \cdot \rho\left(y_{1}\right)\right) \cdot\left(\rho\left(x_{2}\right) \cdot \rho\left(y_{2}\right)\right)=\mu_{\rho}\left(x_{1}, y_{1}\right) \cdot \mu_{\rho}\left(x_{2}, y_{2}\right)$

Hence, $\mu_{\rho}$ is fuzzy dot sub algebra of $X * X$. $\square$

Definition 3.10: Let $\rho$ be a fuzzy subset of $X$. A fuzzy relation $\mu$ on $X$ is called a fuzzy $\rho$-product relation $\mu_{\rho}(x, y) \geq \rho(x) . \rho(y)$ for $x, y \in X$

Let $\rho$ be a fuzzy subset of $X$,A fuzzy relation $\mu$ on $X$ is called a left fuzzy relation $\mu_{\rho}(x, y)=\rho(x)$ for $x, y \in X$

Similarly we can define a right fuzzy relation on $\rho, \mu_{\rho}(x, y)=\rho(y)$ for $x, y \in X$.

Note that a left (respectively, right) fuzzy relation on is a $\rho$-product relation.

Theorem 3.10: Let $\mu$ be a left fuzzy relation on a fuzzy subset $\mu$ of $X$.If $\mu$ is fuzzy dot sub algebra of $X \times X$,then $r h o$ is fuzzy dot sub algebra of $X$.

Theorem 3.11:

Let $\mu$ be a fuzzy relation on $X$ satisfying the inequality $\mu(x, y) \leq \mu(x, 0)$ for all $x, y \in X$, Given $s \in S$, let $\rho_{s}$ be a fuzzy subset of $X$,defined by $\rho_{s}(x)=\mu(x, s)$, for all $x \in X$. If $\mu$ is a fuzzy dot sub algebra of $X \times X$, then $\rho_{s}$ is a fuzzy dot sub algebra of $X$,for all $s \in X$.

Proof. Let $x, y, s \in X$, Then

$\rho_{s}(x * y)=\mu(x * y, s)$

$\mu(x * y, s)=\mu(x * y, s * 0)$

$\mu(x * y, s * 0)=\mu(x, s) *(y, 0)$

$\mu(x, s) *(y, 0) \geq \mu(x, s) \cdot \mu(y, 0)$

$\mu(x, s) \cdot \mu(y, 0) \geq \mu(x, s) \cdot \mu(y, s)$

$\mu(x, s) \cdot \mu(y, s)=\rho_{s}(x) \cdot \rho_{s}(y)$

Therefore, $\rho_{s}$ is fuzzy dot sub algebra of $X$. $\square$

Theorem 3.12:

Let $\mu$ be a fuzzy relation on $X$ and let $\rho_{\mu}$ be a fuzzy sub set of $X$ given by $\rho_{\mu}(x)=$ $i n f_{y \in X}\{\mu(x, y), \mu(y, x)\}$ for all $x \in X$. If $\mu$ is a fuzzy dot sub algebra $X \times X$ satisfying the equality $\mu(x, 0)=1=\mu(0, x)$ for all $x \in$, then $\rho_{\mu}$ is a fuzzy dot sub algebra of $X$.

Proof. Let $x, y, z \in X$, we have

$\mu(x * y, z)=\mu(x * y, z * 0)$

$\mu(x * y, z * 0)=\mu((x, z) *(y, 0))$

$\mu((x, z) *(y, 0)) \geq \mu(x, z) \cdot \mu(y, 0)$

$\mu(x, z) \cdot \mu(y, 0)=\mu(x, z)$,

$\mu(z, x * y)=\mu(z * 0, x * y)$

$\mu(z * 0, x * y)=\mu((z, x) *(0, y))$

$\mu((z, x) *(0, y)) \geq \mu(z, x) \cdot \mu(0, y)$

$\mu(z, x) \cdot \mu(0, y)=\mu(z, x)$, It follows that $\mu(x * y, z) \cdot \mu(z, x * y) \geq \mu(x, z) \cdot \mu(z, x)$

$\mu(x, z) \cdot \mu(z, x) \geq(\mu(x, z) \cdot \mu(z, x)) \cdot(\mu(y, z) \cdot \mu(z, y))$

So that $\rho_{\mu}(x * y)=i n f_{z \in X} \mu(x * y, z) \cdot \mu(z, x * y)$

$i n f_{z \in X} \mu(x \quad * \quad y, z) \cdot \mu(z, x \quad * \quad y) \quad=$

$\left(i n f_{z \in X} \mu(x, z) \cdot \mu(z, x)\right) \cdot\left(i n f_{z \in X} \mu(y, z) \cdot \mu(z, y)\right)$

$\left(i n f_{z \in X} \mu(x, z) \cdot \mu(z, x)\right) \cdot\left(i n f_{z \in X} \mu(y, z) \cdot \mu(z, y)\right)=$ $=\rho_{\mu}(x) \rho_{\mu}(y)$

Therefore $\rho_{\mu}$ is fuzzy dot sub algebra of $X$. $\square$ 
Proposition 3.11: Let $\mathrm{f}$ be a $B F$ - homomorphism from $\mathrm{X}$ into $\mathrm{Y}$ and $\mathrm{G}$ be a fuzzy dot $B F$-subalgebra of $\mathrm{Y}$ with the membership function $\mu_{G}$. Then the inverse image $f^{-1}(G)$ of $\mathrm{G}$ is a fuzzy dot $B F$ - subalgebra of $\mathrm{X}$.

Proof. Let $f: X \rightarrow Y$ and $\mathrm{G}$ be a fuzzy dot $B F$-subalgebra of $\mathrm{Y}$ and let $x, y \in X$. Then

$$
\begin{array}{r}
\mu_{f^{-1}(G)}(x \star y)=\mu_{G}(f(x \star y)) \\
=\mu_{G}(f(x) \star f(y)) \\
\geq \min \left\{\mu_{G}(f(x)), \mu_{G}(f(y))\right\} \\
\geq \mu_{G}(f(x)) \cdot \mu_{G}(f(y)) \\
=\mu_{f^{-1}(G)}(x) \cdot \mu_{f^{-1}(G)}(y) .
\end{array}
$$

Hence $f^{-1}(G)$ isafuzzydotBF - subalgebraof $X$.

Proposition 3.12: Let f be a $B F$-homomorphism from $\mathrm{X}$ onto $\mathrm{Y}$ and $\mathrm{D}$ be a fuzzy $B F$-subalgebra of $\mathrm{X}$ with the suppropery. Then the image $f(D)$ of $\mathrm{D}$ is a fuzzy dot $B F$-subalgebra of $\mathrm{Y}$.

Proof. Let $\mathrm{f}$ be a $B F$-homomorphism fron $\mathrm{X}$ into $\mathrm{Y}$ and let $\mathrm{D}$ be a fuzzy dot $B F$-subalgebra of $\mathrm{Y}$ with supproperty and let $a, b \in Y$, let $x_{0} \in f^{-1}(a), y_{0} \in f^{-1}(b)$ such that $\mu_{D}\left(x_{0}\right)=$ $\sup _{D}(t)_{t \in f^{-1}(a)}, \mu_{D}\left(y_{0}\right)=\sup _{D}(t)_{t \in f^{-1}(b)}$. Then by the definition $\mu_{f(D)}$, we have

$$
\begin{array}{r}
\mu_{f(D)}(x \star y)=\sup _{D}(t)_{t \in f^{-1}(a \star b)} \\
\geq \mu_{D}\left(x_{0} \star y_{0}\right) \\
\geq \min \left\{\mu_{D}\left(x_{0}\right), \mu_{D}\left(y_{0}\right)\right\} \\
\geq \mu_{D}\left(x_{0}\right) \cdot \mu_{D}\left(y_{0}\right) . \\
\text { Consequently } \mu_{f(D)}(x \star y) \geq \mu_{D}\left(x_{0}\right) \cdot \mu_{D}\left(y_{0}\right) \\
=\sup _{D}(t)_{t \in f^{-1}(a) \text {.sup } \mu_{D}(t)_{t \in f^{-1}(b)}=\mu_{f(D)}(a) \cdot \mu_{f(D)}(b) .} \text { Hence } \mu_{f(D)}(x \star y) \geq \mu_{f(D)}(a) \cdot \mu_{f(D)}(b) .
\end{array}
$$

Thus $f(D)$ is a fuzzy dot BF-subalgebra of $X$.

\section{CONCLUSION}

The concepts of structure of fuzzy dot BF-Sub algebras has been introduced. The fuzzy dot product of BF-sub algebra,Fuzzy dot ideal of BF-sub algebra, $\rho$ product of Fuzzy BF-subalgebras have been discussed. Finally different characterization of Fuzzy dot BFsubalgebras have been investigated.

\section{ACKNOWLEDGMENT}

The authors Thanks to Wollo University for the support in the preparation of the paper and the referees for their valuble comments.

\section{REFERENCES}

[1] Andrzej Walendziak,On BF-algebras,Mathematica Slovaca.,2007.,Vol.57.,119-128,http://dmlcz/dmlcz/136941001,Dol:10,2478/s12175-007-003-x.

[2] Barbhuiya,S.R.,Doubt fuzzy ideals of BF-algebra,IOSR Journal of Mathematics.,2014.,Vol.10.,65-60.

[3] Borumand Saeid,A. and Rezvani,M.A.,On Fuzzy BFAlgebras,International Mathematical Forum.,2009.,Vol.1., 13 25.

[4] Jie Meng, Young one Jun and Hee Sij Kim, Fuzzy implicative ideals of BCK-algebras, Fuzzy sets and systems ,vol.89(1997),243-248.

[5] Neggers, J. and Kim, H.S.,On B-algebras, Math.vensik 54(2002), 21-29.

[6] Rosen field, A. Fuzzy Groups, J.Math. Anal. Appl., vo.35(1971),512-517.

[7] Sung Miko, Structure of BF-algebra , Applied Mathematical Sciences,Vo.9, 2015,no.128, 6369-6374, http://dx-doiorg/10,12988/ams.2015,58556.

[8] Zadeh,L.A., Fuzzy sets, Inform and Control.,1965.,vol. 8.,338353.

Creative Commons Attribution License 4.0 (Attribution 4.0 International, CC BY 4.0)

This article is published under the terms of the Creative Commons Attribution License 4.0

https://creativecommons.org/licenses/by/4.0/deed.en_US 